\title{
Can Fuzzy Logic Bring Complex Problems into Focus? Modeling Imprecise Factors in Environmental Policy
}

\author{
Thomas E. McKone* and Ashok W. Deshpande** \\ *University of California, Berkeley \\ ${ }^{* *}$ Former Deputy Director, National Environmental Engineering Research Institute, \\ India
}

In modeling complex environmental problems, we often fail to make precise statements about inputs and outcome. In this case the fuzzy logic method native to the human mind provides a useful way to get at these problems.

\section{Introduction}

You and a friend walk outside on an April morning. You announce that the weather is "mild". Your friend declares it "cold". Who is wrong? Or are you both right? We all recognize that language can be imprecise and that words such as cold, hot, or mild do not have well-defined boundaries. In 1965, Lotfi Zadeh introduced fuzzy logic as a means of processing data by extending classical set theory to handle partial membership1. Classical set theory deals with sets that are "crisp" in the sense that members are either in or out according to rules of binary logic. For example the apple in the basket is Red OR Not Red (binary logic). Some of the apples could be categorized as Red AND Not Red (fuzzy logic). Many of the concepts that we deal with in everyday life and in fields such environmental health involve factors that defy classification into "crisp" sets-safe, harmful, acceptable, unacceptable, etc. A classic example is when a regulator, who after she carefully explains the result of a detailed quantitative risk assessment to a community group is then asked "But are we safe?" In this case, "safe," defies crisp classification because it is a multivariate state with gradations, that very among different individuals and groups.

Fuzzy logic has become a common way of dealing with information in a number of fields, such as control theory, smart machines, investment analysis and so on. But the application of fuzzy sets can and has been extended to environmental science and policy. For anyone who has worked on health and environmental issues, it becomes immediately obvious that we deal constantly with fuzzy concepts-hazard, acceptable, safe, etc. Even concepts such as carcinogen and neurotoxin define fuzzy sets whose 
members are selected by experts who review and make judgments on conflicting toxicology or epidemiology. In spite of their relevance and early efforts to promote their use in risk assessment ${ }^{2}$, fuzzy logic applications are still rare in risk assessment or other environmental assessments.

In this paper we consider whether and how fuzzy logic and fuzzy arithmetic apply to risk assessment and environmental policy. We use a case study assessment of water quality in the Ganga river of India to illustrate this evaluation. Our goal is to consider whether and how much this approach can be applied more broadly for environmental assessments.

\section{Fuzzy Sets, Fuzzy Logic, and Fuzzy Arithmetic}

Fuzzy concepts come largely from the field of soft computing and have links to many earlier influences, among them Zadeh's 1965 paper on fuzzy sets ${ }^{1}$ and his paper on the analysis of complex systems and decision processes ${ }^{3}$. Confronting fuzzy concepts requires three skills-(1) the ability to construct fuzzy sets (those with partial membership) and the ability to perform (2) logical operations and (3) arithmetic operations on those sets. Each of these capabilities must be employed to carry out our case study. We introduce them briefly here.

\section{Fuzzy Sets}

In contrast to classical sets, fuzzy sets include objects with partial membership. Some view a person whose age is 45 as "old" and others as "young". So this person's age has partial membership in both the fuzzy set "old" and the fuzzy set "young". The process of defining membership produces a "membership" function for fuzzy sets. This is illustrated in Figure 1 where we show example membership functions in the linguistic sets "cold", "mild", or "hot" for a range of temperatures. In the language of fuzzy sets this figure represents functions $\mu_{A}$ that express the degree of membership of elements $x$ (temperatures) in the set $A$, where $A=$ cold, mild, or hot. $\mu_{A}$ is a set of ordered pairs where the first element of the ordered pair is from the set $x$ of temperatures and the second element from the interval $[0,1]$ that expresses membership in $A$. Here 0 represents non-membership, 1 represents complete 
membership, and values in between represent intermediate degrees of membership. We can determine membership in a fuzzy set either by observation or by eliciting characterizations from experts or users.

At this point it is useful to make a distinction between fuzzy logic and probability theory, a distinction that involves the difference between the notions of probability and a degree of membership. In contrast to probability density functions, fuzzy membership functions express the possibility of an outcome rather than its probability. Probability statements are about the likelihood of outcomes. In a probabilistic approach, we model uncertainty by expressing our belief that an event either occurs or does not. But with fuzzy logic we model uncertainty as the degree of membership in set that defines an outcome.

\section{Fuzzy Logic}

Fuzzy logic is the key to building models of fuzzy systems by providing rules for operations on fuzzy sets. The first thing to recognize is that fuzzy logic is a generalization of standard Boolean logic. In other words, if we keep the fuzzy values at their extremes of 1 (completely true), and 0 (completely false), fuzzy logic reduces to standard binary logic.

Three basic operations apply to fuzzy sets-negation, intersection, and union. To negate a fuzzy set, one simply subtracts the membership value in the fuzzy set from 1. For example, in Figure 1 the membership value in "cold" at $5{ }^{\circ} \mathrm{C}$ is 1 . With negation, the membership value at $5{ }^{\circ} \mathrm{C}$ becomes 0 . The intersection of two sets is the minimum of the two membership values at each point on the x-axis. In Figure 1 the set "cold" has a membership of 0.7 corresponding to $x=14^{\circ} \mathrm{C}$ and the fuzzy set "mild" has a membership of 0.3 corresponding to $x=14^{\circ} \mathrm{C}$. The intersection has a membership value of 0.3 at $x=14^{\circ} \mathrm{C}$. The union of two sets is the maximum of the two membership values at each point on the x-axis. Referring again to Figure 1, the union of the sets "cold" and "mild" at $x=14^{\circ} \mathrm{C}$ has membership 0.7. In mathematical terms, we have: 
Negation: $\quad \mu_{\operatorname{not} A}(x)=1-\mu_{A}(x)$

Intersection: $\quad \mu_{A \cap B}(\mathrm{x})=\operatorname{Min}\left[\mu_{\mathrm{A}}(x), \mu_{B}(x)\right]$

Union: $\quad \mu_{A \cup B}(\mathrm{x})=\operatorname{Max}\left[\mu_{A}(x), \mu_{B}(x)\right]$

For fuzzy sets, there are extensions of these standard set operations (union, intersection, etc.) and fuzzy sets operators that are uniquely fuzzy operations with no counterpart in ordinary set theory. For example there are families of functions such as the triangular norm (t-norm) for intersection and the t-conorm (or s-norm) for unions that introduce different options for binary mapping to aggregate two membership functions. Operations that are unique to fuzzy sets also include concentration, dilation, normalization, intensification, fuzzification. These operators leave classical (crisp) sets unchanged. The concentration operation compresses a membership function and reduces the degree of membership of marginal members by squaring the degree of membership of each member in the set. The dilation operator expands the membership function of the peripheral members by taking the positive square root of the degree of the membership of each set element. Normalization modifies the membership value of all elements by the factor needed to increase the membership status of at least one member to a maximum of 1 . Intensification makes a fuzzy set less fuzzy by increasing by some defined factor the degree of membership of all set elements that have membership greater than 0.5 and decreasing the degree of membership of elements with membership less than 0.5. Fuzzification operates in reverse of intensification and makes the set fuzzier.

\section{Fuzzy Arithmetic}

In addition to logic operations, there are interval arithmetic operations that apply to fuzzy sets. Fuzzy arithmetic provides the foundation for possibility theory introduced by Zadeh ${ }^{3}$ and further developed by Kaufmann and Gupta ${ }^{4}$ and Dubois and Prade $^{5}$. Although it is analogous to probability theory, possibility theory can be carried out under weaker assumptions and thus used when limited experimental or observational information is available. 
Among the important arithmetic operations on fuzzy sets are addition, subtraction, multiplication, division, and degree of match (DM), which we define and use for the example below. Addition produces a set with an interval range that includes all of the sums of the addends of the two sets being added. For example, if we add numbers from set $A$ in the range $[1,5]$ with numbers from set $B$ in the range $[2,4]$, we obtain a set of numbers $C$ in the range $[3,9]$. Members, $x$, of set $A$ with membership function $\mu_{A}(x)$ and members, $y$, of set $B$ with membership function $\mu_{B}(y)$, produce elements, $z=x+y$, in set $C$ that have membership $\mu_{C}(z)$ determined as

$$
\mu_{C}(z)=\min _{\sup z=x+y}\left[\mu_{A}(x), \mu_{B}(y)\right]
$$

Which means that we calculate the degree of membership in $C$ for each pair $x, y$ derived from $\mathrm{A}$ and $\mathrm{B}$ as $\min \left[\mu_{A}(x), \mu_{B}(x)\right]$ then determine $\mu_{C}(z) \mathrm{s}$ the maximum (sup) among all combinations $x=y$ the produce a given $z$ value. A similar approach applies to subtraction, multiplication, and division. The DM operator finds its place in fuzzy-rule based systems. DM is the measure of overlap in the membership functions of two fuzzy sets. For arbitrary sets $\mathrm{A}$ and $\mathrm{B}, \mathrm{DM}(A, B)$ is defined as:

$$
D M(A, B)=\frac{\int \mu_{A \cap B}(x) d x}{\int \mu_{A}(x) d x}
$$

in which $x$ denotes the range of parameter values (dissolved oxygen, fecal coliform level, etc) and $\mu_{A \cap B}(\mathrm{x})$ is the membership function for the intersection of fuzzy sets $A$ and $B$.

\section{How it Works: Bathing Water Quality in the Ganga River}

We illustrate the use of fuzzy logic in environmental health assessment with fuzzy descriptions of bathing water quality in the river Ganga, India. When Ganga water quality progressively deteriorated due to indiscriminate discharge of municipal sewage and industrial effluents, Deshpande et al. $^{6}$ used fuzzy classification to characterize water quality after the implementation of pollution control measures. Ceremonial bathing plays a vital role in cultural, social, and religious life of India. The process of defining 
water quality for bathing involves a great deal of uncertainty. We focus on the acceptability of water for bathing at two sampling stations, Rishikesh on the upstream of the river and Varanasi where the pollution is severe. Both places are important for religious bathing, but Varanasi is of particular importance as tens of thousands of people take a religious bath daily at defined ghats (bathing places).

This example presents modeling of both the statistical uncertainty in the field data and cognitive uncertainty in judgments of experts. Water quality experts have identified five parameters for defining river water quality for bathing-fecal coliform (FC), dissolved oxygen (DO), biochemical oxygen demand (BOD), $\mathrm{pH}$ and turbidity. Measured values of these parameters must communicate river water quality to policy makers and the public at large. But how? Observed values of these parameters are uncertain due to measurement error and natural variability. Quantitative risk assessment is precluded by the absence of adequate exposure and dose-response models for each of these five parameters. Another approach to communicate water quality is an aggregated water quality index (WQI) expressed in numbers ranging between 0 and 100. If WQI is less than 20, the water quality is considered bad and so on. One problem with this type of highly subjective approach, is that final score fails to communicate the uncertainty in (a) the measurement of these factors, (b) the interpretation of an acceptable range for each parameter, and (c) the method used to integrate these dissimilar factors. Neither risk assessment nor WQI can signify the degree of certainty attached to any linguistic description of results. So we introduce fuzzy logic to characterize water quality in a way that provides linguistic terms (highly accepted,...,not accepted) with quantified degrees of certainty. The result is an alternative approach with more fidelity to the type uncertainties involved in this particular problem.

This is but one example of a fuzzy logic application to environmental quality. There or others in the current literature. For example Regan et al. ${ }^{7}$ have demonstrated the advantages of using fuzzified definitions of "endangered" in conservation biology. 


\section{Matching Fuzzy Values}

We construct fuzzy sets to represent in linguistic terms both field observations on parameter values and judgments of experts about acceptability of each parameter value range. The degrees of match between observations and fuzzy terms relating to acceptability are used to establish overall water quality. This process is illustrated in Figure 2. First, we fit the measured parameter to a convex normalized fuzzy-interval as shown in the top diagram of Figure 2. The membership function here represents the range of a given water quality parameter, such as fecal coliform level. We next use expert elicitation to construct fuzzy sets for modeling expert opinion in classifying a specific factor, such as fecal coliform level, as being "very good", "good", "fair" or "poor". The values for which all experts assign the same value are given membership 1.0 and the values for which no expert assigns that term are given membership 0.0. We derive values of the membership functions in between by connecting the 0 and 1 membership values with a continuous straight line. This is shown in the middle diagram of Figure 2. We use the DM operator (described above) to determine the degree of match between the convex normalized fuzzy numbers describing observed parameter value ranges and the fuzzy sets describing the experts' quality classification ranges. This is shown at the bottom of Figure 2. Table 1 provides the results of this process with the DM values at Rishikesh and Varanasi for each linguistic class. The numerator for each DM is derived from the fuzzy interval corresponding to the experts' water quality classification.

\section{Fuzzy Rule Based System}

We construct rules that classify water based on the status of the quality parameters as expressed by DM. Figure 3 provides the flow chart used to develop these rules and three primary attributes to classify quality-bacteriological status, biochemical status, and physical status. Bacteriological status is linked to fecal coliform levels and physical status is linked to turbidity, but biochemical status is linked to three secondary attributes-DO, BOD, and $\mathrm{pH}$. At each stage of this hierarchical structure, we apply the experts' acceptability rules in order to classify water quality and obtain a degree of certainty about the classification. Examples of these rules at each stage and the corresponding DM for Varanasi (in parentheses) are: 
At the first stage

If $\mathrm{DO}$ is $<\operatorname{good}>(\mathbf{0 . 1 6})$ and

BOD is < very good $>(\mathbf{0 . 1 3})$ and

$\mathrm{pH}$ is $<\operatorname{good}>(\mathbf{0 . 3 0})$

Then biochemical status of water will be $<$ good $>(\mathbf{0 . 1 3})$.

At the second stage

If Bacteriological status of water is $<\operatorname{good}>(\mathbf{0 . 8})$ and

Biochemical status of water is $<\operatorname{good}>(\mathbf{0 . 1 3})$ and

Physical status is < very good $>(\mathbf{0 . 9 8})$

Then the water quality for bathing is < accepted $>(\mathbf{0 . 1 3})$

Of course there are many rules of this type needed to specify our water quality systems. One of the challenges of fuzzy applications is checking the reasonableness of all these rules. As we see from the example above, the conjunctive (intersection) operator applies when we want to find the DM of one or more antecedent rules, that is

$\mathrm{DM}$ (of a rule with steps $1,2,3 \ldots \mathrm{n})=\mathrm{Min}\left(\mathrm{DM}_{1}, \mathrm{DM}_{2}, \mathrm{DM}_{3}, \ldots \mathrm{DM}_{\mathrm{n}}\right)$

We must also address the DM for cases of "disjuncts." Disjuncts are different rules that lead to the same classification. For example at the right of Figure 3 there can be multiple rules that result in the classification of water as "accepted". In this case we apply the disjunctive (union) operator:

$\mathrm{DM}$ (of disjuncts $1,2, \ldots \mathrm{n})=\operatorname{Max}\left(\mathrm{DM}_{\text {disjunct1 }}, \mathrm{DM}_{\text {disjunct2}}, \ldots \mathrm{DM}_{\text {disjunct1 }}\right)$

The lower part of Table 1 describes at Rishekesh and Varanasi the degree of certainty for each linguistic classification that we obtained by using the DMs from the upper part of the table and with a set of acceptance rules. We note here that at Rishekesh the water quality is "accepted" with certainty 0.41 . The next certainty value of 0.36 for "just accepted" indicates that the outcome is more towards "just accepted" than towards "highly accepted". Water at Varanasi is "not accepted" with degree of certainty 0.98. 
This indicates an urgent need to intensify the pollution control efforts along the river Ganga with focus on the reduction on bacterial counts. In following this example, the reader must recognize our goal here is to present only the inputs, intermediate steps, and results of the analysis. Many of the essential details could not be presented in this type of summary paper.

\section{The Outlook for Fuzzy Logic in Environmental Policy}

Over the last few decades, soft computing tools such fuzzy logic based methods, neural networks, and genetic algorithms have had significant and growing impacts. But we have seen only limited use of these methods in environmental fields such as risk assessment, cost-benefit analysis, and life-cycle impact assessment. Because fuzzy methods offer both new opportunities and unforeseen problems relative to current methods, it is difficult to determine how much impact such methods will have on environmental policies in the coming decades. Here we consider some obvious advantages and limitations.

Quantitative models with explicit and crisp delineations of systems have long been the currency of discourse in engineering and the physical sciences, where basic physical laws form the foundations of analyses. These fields place high value on the causal linkages implicit in model structure and parameterization. But problems that involve human values, language, control theory, biology, and even environmental systems have had to rely more on descriptive and empirical approaches ${ }^{8}$. In these latter fields our goal is often to summarize the observations in an efficient and useful manner. For these important areas of science and health, fuzzy logic based methods should be further investigated as alternative and perhaps more appropriate methods to confront uncertain and complex systems.

For the types of complex and imprecise problems that arise in environmental policy, the ability to model complex behaviors as a collection of simple if-then rules makes fuzzy logic an appropriate modeling tool. Because fuzzy arithmetic works well for addressing linguistic variables and poorly characterized parameters, fuzzy methods offer the opportunity to evaluate and communicate assessments based in linguistic terms that could possibly match those of the public and decision makers. Moreover 
approximate reasoning methods such as fuzzy arithmetic do not require wellcharacterized statistical distributions as inputs. Another key advantage of fuzzy logic in risk assessment is the ability to merge multiple objectives with different values and meaning, for example combining health objectives with esthetic objectives. It also provides rules for combining qualitative with quantitative objectives.

But we must recognize and confront the potential limitations of fuzzy logic for expressing health risk and other environmental impacts. One problem is the strong reliance on subjective inputs. Although this is a problem in any type of impact assessment, fuzzy methods might provide more opportunity to abuse the use of subjective inputs. Moreover, although well suited to addressing uncertainty (lack of knowledge), some argue that for addressing variability (heterogeneity) fuzzy logic has not been shown as superior to standard statistical descriptions. Ferson and Tucker have noted that that, because it can fail to capture the value range of complex data sets and the correlations among parameters, fuzzy arithmetic may not be appropriate for routine use in risk assessments concerned primarily with variability ${ }^{9}$. However, Ozbek and Pinder (2003a) have addressed both variability and uncertainty in a fuzzy logic approach to the risk equations in a groundwater remediation problem. ${ }^{10}$ They use statements and preferences of practicing toxicologists to construct fuzzy rules and relate these rules to relate a pattern of exposure of benzene to its carcinogenic effects. Rules describing individual susceptibility address parameters typically represented by variability in other risk assessments. The rules constrain the risk model in a way that preserves interindividual differences.

Although probabilistic assessments based on tools such as Monte Carlo are analogous to assessments based on fuzzy logic, these two methods differ significantly both in approach and interpretation of results. One key advantage of fuzzy logic over Monte Carlo methods is the ability to confront linguistic variables (safe, unsafe, acceptable, unacceptable). With Monte Carlo methods, we must often force continuous distributions to fit linguistic variables for probabilistic assessments. Fuzzy arithmetic combines outcomes from different sets in a way that is analogous to but different from Monte Carlo methods. Possibility theory can be used as an alternative to probabilistic 
analysis, but this creates the potential for interpreting membership functions as probability distributions.

\section{Concluding Points}

Fuzzy logic represents a significant change in both the approach to and outcome of environmental evaluations. Risk assessment is currently based on the implicit premise that probability theory provides the necessary and sufficient tools for dealing with uncertainty and variability. The key advantage of fuzzy methods is the way they reflect the human mind in its remarkable ability to store and process information which is consistently imprecise, uncertain, and resistant to classification. Our case study illustrates the ability of fuzzy logic to integrate statistical measurements with imprecise health goals. But we submit that fuzzy logic and probability theory are complementary and not competitive.

In the world of soft computing, fuzzy logic has been widely used and has often been the "smart" behind smart machines. But it will require more effort and case studies to establish its niche in risk assessment or other types of impact assessment. Although we often hear complaints about "bright lines," could we adapt to a system that relaxes these lines to fuzzy gradations? Would decision makers and the public accept expressions of water or air quality goals in linguistic terms with computed degrees of certainty? Resistance is likely. In many regions, such as the US and European Union, it is likely that both decision makers and members of the public are more comfortable with our current system in which government agencies avoid confronting uncertainties by setting guidelines that are crisp and often fail to communicate uncertainty. But some day perhaps a more comprehensive approach that includes exposure surveys, toxicological data, epidemiological studies coupled with fuzzy modeling will go a long way in resolving some of the conflict, divisiveness, and controversy in the current regulatory paradigm.

\section{Acknowledgements}

We thank Michael Sohn and an anonymous reviewer for a number of thoughtful review comments. We also thank Metin Ozbek and George Pinder for offering both valuable inputs to and a detailed review of our final draft. This work was supported by 
the US Environmental Protection Agency National Exposure Research Laboratory through Interagency Agreement \# DW-988-38190-01-0 and carried out at Lawrence Berkeley National Laboratory through the US Department of Energy under Contract Grant No. DE-AC03-76SF00098.

\section{References}

[1] Zadeh, Lofti A. "Fuzzy sets." Information and Control 8: 338-353, 1965.

[2] Unwin, S.D. A fuzzy set theoretic foundation for vagueness in uncertainty analysis, Risk Analysis, 6(1):27-34, 1986.

[3] Zadeh, L. Fuzzy sets as a basis for a theory of possibility. Fuzzy Sets and Systems 1:3-28, 1978.

[4] Kaufmann, A., and Gupta, M.M. Introduction to Fuzzy Arithmetic: Theory and Applications. Van Nostrand Reinhold, New York 1985.

[5] Dubois, D., Prade, H. Possibility Theory: An Approach to Computerized Processing of Uncertainty. Plenum Press, New York, 1988.

[6] Deshpande, A.W., Raje, D.V., Khanna, P. Fuzzy description of river water quality, Proceedings 1994 European Congress on Intelligent Techniques and Soft Computing (EUFIT) September, 1994, Achen, Germany.

[7] Regan, H.M., Colyvan M., Burgman M.A. A proposal for fuzzy International Union for the Conservation of Nature (IUCN) categories and criteria. Biological Conservation, 92(1):101-108, 2000.

[8] Spear, R.C. Mathematical Modeling in Environmental Health, Environmental Health Perspectives 110(7) A382, 2002.

[9] Ferson, S.; Tucker, W. Fuzzy arithmetic does not yield conservative risk assessments, presentation at the annual meeting of the Society for Environmental Toxicology and Chemistry, November, 2002, Salt Lake City, Utah. (A paper on this subject will be published in the forthcoming volume Application of Uncertainty Analysis to Ecological Risks of Pesticides, A. Hart (ed) SETAC press.

[10] Ozbek, M.M. and Pinder, G.F. Risk-based remedial design: modeling and implementation of expert insight, In: Bentley et al. (eds), Computational Methods in Water Resources XIII, Calgary, Canada, pp. 525-531. Balkema, 2000.

[11] Ozbek, M.M. and Pinder, G.F. Water Quality and Health Risk Analysis Using Fuzzy Sets and Fuzzy Logic, Technical Report RCGRD 12-03-1, Department of 
Civil and Environmental Engineering, University of Vermont, 2003. (submitted for publication) 
Table 1 Degree of Match Between Field Data and Fuzzy Terms and the Resulting Fuzzy Description of Water Quality with Degree of Certainty

\begin{tabular}{|c|c|c|c|c|c|}
\hline \multicolumn{6}{|c|}{ Degree of match between parameters and linguistic class } \\
\hline & & \multicolumn{4}{|c|}{ Linguistic class } \\
\hline $\begin{array}{l}\text { Sampling } \\
\text { Location }\end{array}$ & Parameter & Very good & Good & Fair & Poor \\
\hline \multirow[t]{5}{*}{ Rishikesh } & $\begin{array}{l}\text { Fecal } \\
\text { coliform }\end{array}$ & 0.21 & 0.80 & 0.36 & 0 \\
\hline & $\begin{array}{c}\text { Dissolved } \\
\text { Oxygen }\end{array}$ & 0.45 & 0.16 & 0 & 0 \\
\hline & $\mathrm{BOD}$ & 0.13 & 0.58 & 0.40 & 0.02 \\
\hline & $\mathrm{pH}$ & 0.41 & 0.30 & 0.23 & 0.10 \\
\hline & Turbidity & 0.98 & 0.03 & 0 & 0 \\
\hline \multirow[t]{5}{*}{ Varanasi } & $\begin{array}{c}\text { Fecal } \\
\text { coliform }\end{array}$ & 0 & 0 & 0.01 & 0.98 \\
\hline & $\begin{array}{c}\text { Dissolved } \\
\text { Oxygen }\end{array}$ & 0.16 & 0 & 0 & 0 \\
\hline & BOD & 0.50 & 0.33 & 0.12 & 0 \\
\hline & $\mathrm{pH}$ & 0.68 & 0.47 & 0.29 & 0.06 \\
\hline & Turbidity & 0.68 & 0.51 & 0 & 0 \\
\hline \multicolumn{6}{|c|}{ Description of water quality with degree of certainty } \\
\hline & & & \multicolumn{3}{|c|}{ City } \\
\hline \multicolumn{2}{|c|}{ Water quality descriptior } & \multicolumn{2}{|r|}{ Rishikesh } & \multicolumn{2}{|c|}{ Varanasi } \\
\hline \multicolumn{2}{|c|}{ Highly accepted } & \multicolumn{2}{|r|}{0.21} & \multicolumn{2}{|c|}{0} \\
\hline \multicolumn{2}{|c|}{ Accepted } & \multicolumn{2}{|r|}{0.41} & \multicolumn{2}{|c|}{0} \\
\hline \multicolumn{2}{|c|}{ Just accepted } & \multicolumn{2}{|r|}{0.36} & \multicolumn{2}{|c|}{0.01} \\
\hline \multicolumn{2}{|c|}{ Not accepted } & & 0.10 & \multicolumn{2}{|c|}{0.98} \\
\hline
\end{tabular}




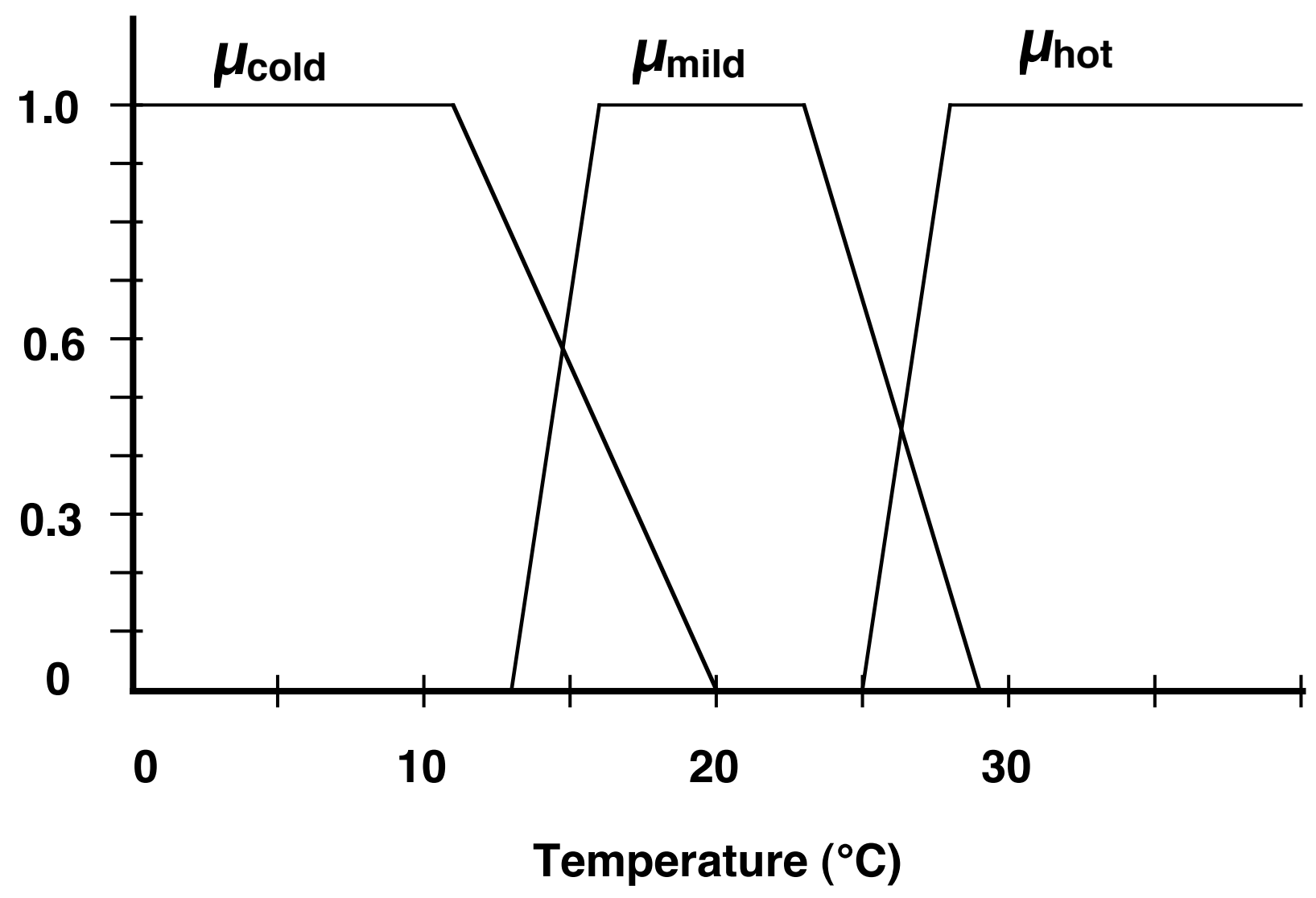

Figure 1. Degree of membership of a range of temperatures in the fuzzy sets "cold", "mild", and "hot". 


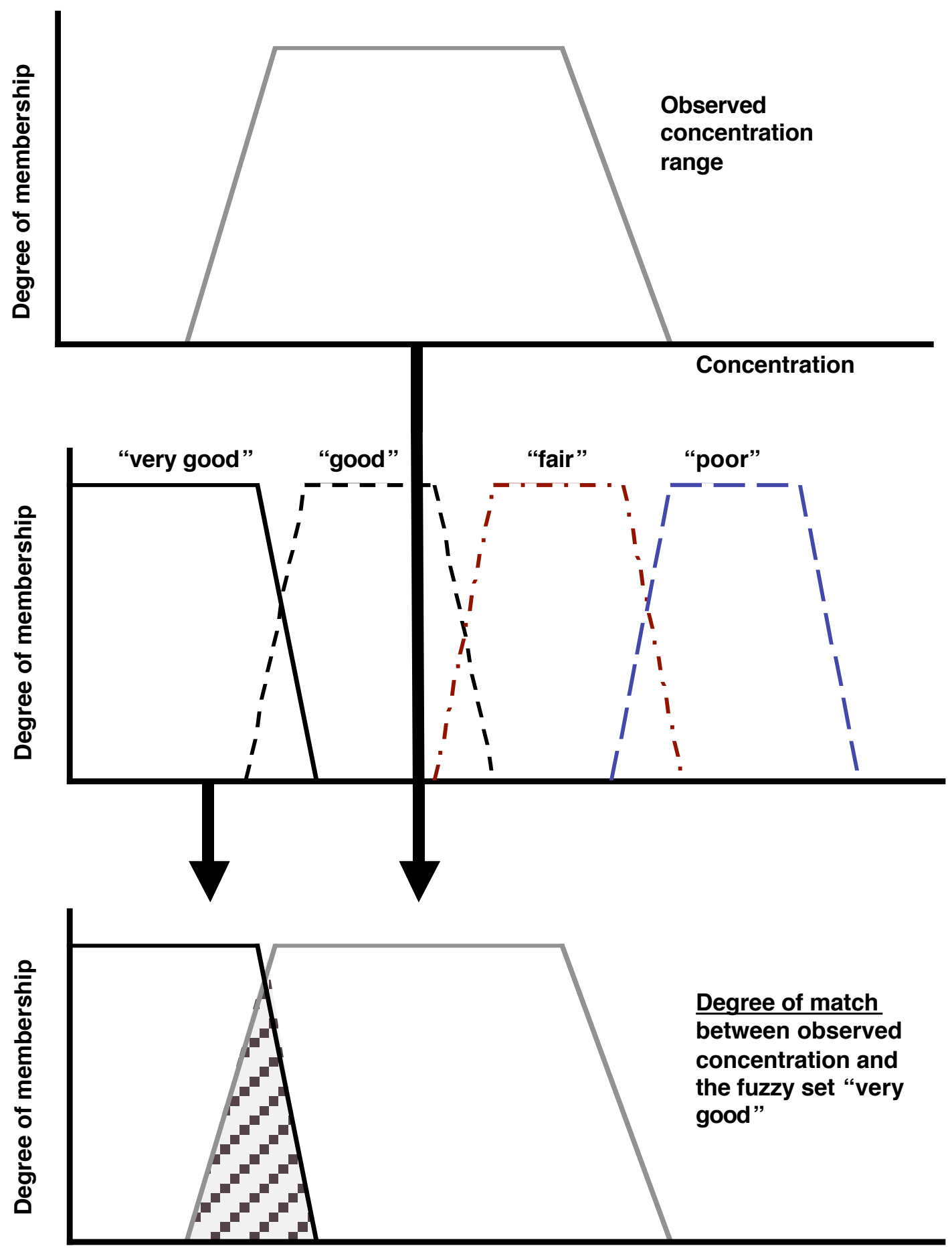

Figure 2. The process for constructing fuzzy numbers and degree of match between the membership functions of observed concentrations and fuzzy terms 


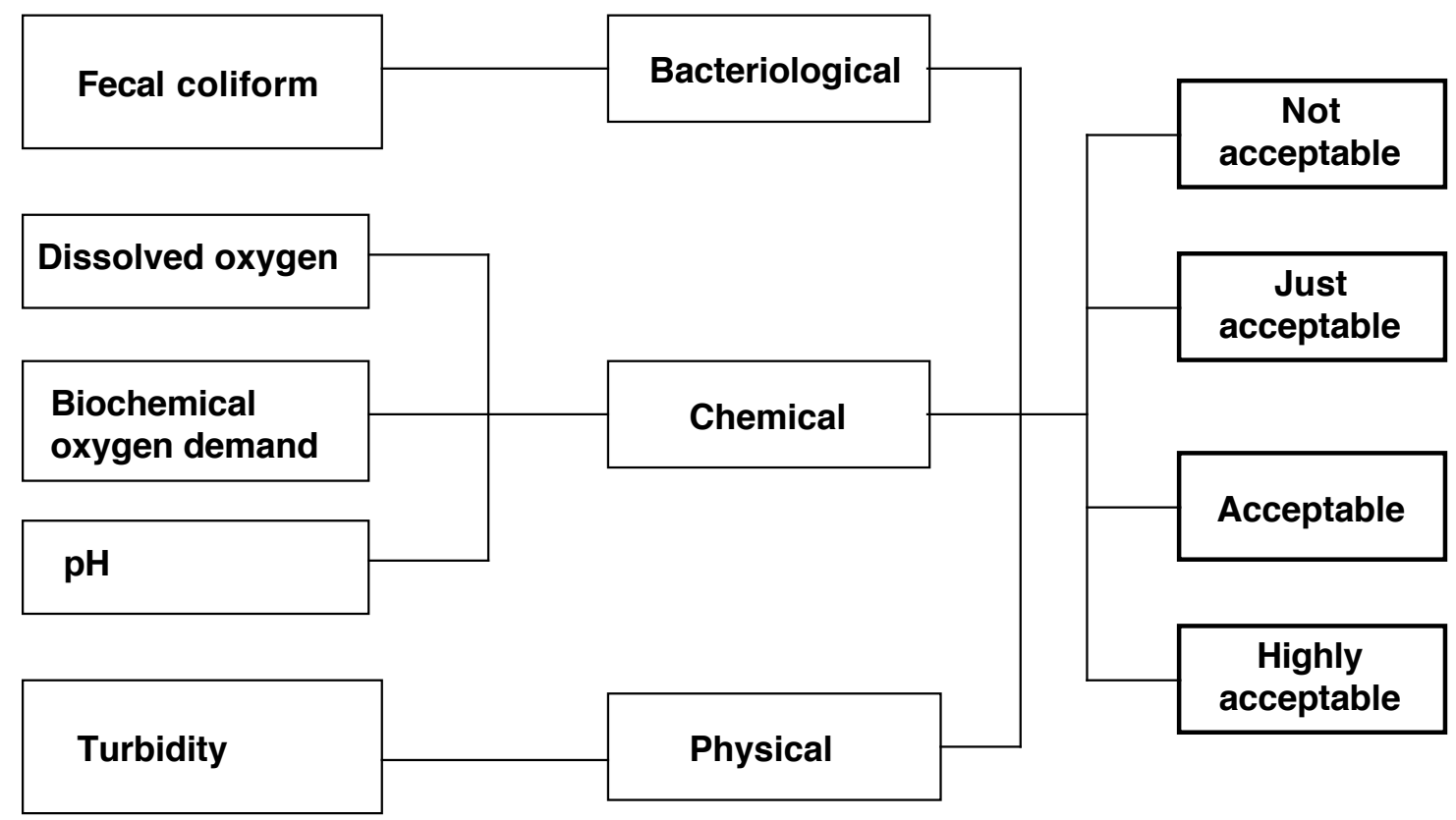

Figure 3. Hierarchical structure for water quality classification. 